\title{
A Case of Extensive Splanchnic Vein Thrombosis in a COVID-19 Patient and a Review of the Literature
}

\author{
Kwang Hwi Lee ${ }^{1}$, Eui Yong Jeon ${ }^{1,{ }^{*}}$, Maryam Hasan Abdulla ${ }^{2}$, Young Joon Ahn ${ }^{3}$, George Thomas ${ }^{3}$ and \\ Yeong-lee $\mathrm{Yu}^{4}$ \\ ${ }^{1}$ Department of Radiology, Sheikh Khalifa Specialty Hospital, Ras al Khaimah, United Arab Emirates \\ ${ }^{2}$ Department of Medical Oncology, Sheikh Khalifa Specialty Hospital, Ras al Khaimah, United Arab Emirates \\ ${ }^{3}$ Department of Surgical Oncology, Sheikh Khalifa Specialty Hospital, Ras al Khaimah, United Arab Emirates \\ ${ }^{4}$ Department of Pediatrics, Jiahui International Hospital, Shanghai, China \\ "Corresponding author: Department of Radiology, Sheikh Khalifa Specialty Hospital, Ras al Khaimah, United Arab Emirates. Email: euiyong.jeon@sksh.ae
}

Received 2021 March 04; Revised 2021 November 21; Accepted 2021 November 27.

\begin{abstract}
Splanchnic vein thrombosis is one of the rare complications of coronavirus disease 2019 (COVID-19). A 43-year-old woman presented with splanchnic vein thrombosis as a rare extrapulmonary complication of COVID-19. She was previously healthy without a medical history of coagulopathy before hospital admission. She complained of epigastric pain, along with nausea and vomiting. Enhanced abdominopelvic computed tomography (CT) scan demonstrated extensive acute thrombosis in the portal, superior mesenteric, and splenic veins with total occlusion. Intestinal ischemia or infarction was not clinically observed. All thrombophilia screening tests yielded negative results. Under anticoagulation therapy, she recovered dramatically and was discharged from the hospital. Imaging findings can be used to confirm splanchnic vein thrombosis when a COVID-19 patient has abdominal symptoms.
\end{abstract}

Keywords: Color Doppler Ultrasonography, Computed Tomography (CT), COVID-19, Splanchnic Vein Thrombosis

\section{Introduction}

A relatively high incidence of hypercoagulability has been reported in patients with coronavirus disease 2019 (COVID-19). Venous thromboembolism may occur in $13 \%$ of COVID-19 patients due to the imbalance of procoagulant and anticoagulant systems, including 31\% of COVID-19 patients admitted to the intensive care unit (ICU) and 7\% of non-ICU patients (1). Although several reports of isolated splanchnic vein thrombosis have been published in the literature, most cases are critically ill or have a risk factor for hypercoagulability $(2,3)$. Herein, we present the case of a young woman with COVID-19 and isolated splanchnic venous thrombosis, without a history of risk factors for hypercoagulability in the Middle East.

\section{Case Presentation}

A previously healthy, 43-year-old woman with no medical conditions other than anemia was admitted to our hospital. She had experienced epigastric pain (pain scale, 3/10; colicky and sometimes radiating to the back) for 2 weeks, along with nausea and vomiting. She had fever, chills, and dry cough 10 days before admission, but had no subjective symptoms, such as breathing difficulties, chest pain, or changes in bowel habits. She denied smoking or alcohol consumption. She was married and did not use any oral contraceptive pills.

On admission, she was hemodynamically stable and afebrile, without jaundice. The physical examination of the chest and cardiovascular system was unremarkable. Also, in the physical examination of the abdomen, it was found to be soft with mild tenderness over the epigastric area, without organomegaly or palpable lymphadenopathy. In the laboratory tests, she was positive for severe acute respiratory syndrome coronavirus 2 (SARS-CoV-2), based on reverse transcription-polymerase chain reaction (RT-PCR).

The blood tests revealed increased leukocytes (12,000 $\mu \mathrm{L}$; normal range: 4,000 - 10,000 $\mu \mathrm{L})$, decreased hemoglobin (7.7 g/dL; normal range: 12.0 - $16.0 \mathrm{~g} / \mathrm{dL}$ ), decreased hematocrit (24.9\%; normal range: $36-46 \%$ ), increased platelet count $(544 \times 103 \mu \mathrm{L}$; normal range: $130-400 \times 103 \mu \mathrm{L})$, prolonged prothrombin time $(14.4$ sec; normal range: 10.5 - $12.5 \mathrm{sec})$, increased international normalized ratio (1.3; normal range: 0.8 - 1.2), and increased D-dimer level (> $35.20 \mu \mathrm{g} / \mathrm{mL}$; normal range: 0.00 
- $0.50 \mu \mathrm{g} / \mathrm{mL})$. The serum iron level was low $(10 \mu \mathrm{g} / \mathrm{dL}$; normal range: $33-193 \mu \mathrm{g} / \mathrm{dL}$ ). Iron deficiency anemia was diagnosed in the patient. The serum electrolyte tests were unremarkable. Also, rheumatoid factors and other immunological immunoassays showed normal ranges.

Enhanced abdominopelvic computed tomography (CT) scan revealed extensive thrombosis with intraluminal dilatation, and vascular wall enhancement was observed in the portal, splenic, and mesenteric veins with minimal ascites. Nonetheless, significant wall thickening or intramural gas in the bowel was not found (Figures $1 \mathrm{~A}$ \& 1B). Several small subpleural lesions with ground glass attenuation were observed in the right lung on the chest CT scan, suggesting COVID-19 pneumonia (Figure 1C).

The patient was examined by a surgical team closely. The hematology team prescribed an anticoagulation therapy with enoxaparin sodium $(1 \mathrm{mg} / \mathrm{kg}$, subcutaneously twice daily). A thrombophilia workup, including antiphospholipid antibodies, paroxysmal nocturnal hemoglobinuria, factor V Leiden mutation analysis, and factor II (prothrombin) mutation analysis, was also performed. All thrombophilia studies yielded negative results. Moreover, Doppler ultrasonography of the abdomen and lower extremity veins was performed on the third day of admission. It demonstrated no significant change in splanchnic vein thrombosis, while the status of ascites improved, with no evidence of deep vein thrombosis in the lower extremities. After five days of systemic anticoagulation under close observation, her abdominal symptom dramatically improved, and she was discharged under continuous oral anticoagulant therapy.

\section{Discussion}

The clinical manifestations of COVID-19 are mainly related to the respiratory system, ranging from mild symptoms, such as fever, cough, and sputum production, to respiratory distress and respiratory failure (4). However, thromboembolic complications may occasionally occur in COVID-19 patients with extrapulmonary manifestations, such as pulmonary artery thromboembolism, myocardial infarction, and deep vein thrombosis in the lower extremities, with an incidence of 25 to $31 \%$ in critically ill patients treated in the ICU (5).

To the best of our knowledge, splanchnic vein thrombosis in COVID-19 has been rarely reported; in the literature, six cases have been reported so far (2, 3, 6-13). Five patients were Europeans, and one patient was North American. Three of these patients showed isolated thrombosis in the portal vein, which improved with anticoagulant therapy. The other three patients showed more extensive thrombosis in the portomesenteric, hepatic, and splenic veins, and two of them expired.

Unlike the mentioned cases, our patient recovered completely under anticoagulant therapy, although she showed extensive acute thrombosis, involving the main portal vein, splenic vein, and upper-stream mesenteric veins with total occlusion. Our patient is also the first case of COVID-19, presenting with splanchnic vein thrombosis in the Middle East. Besides, she had been previously healthy without any risk factors, although portomesenteric venous thrombosis usually develops in patients with risk factors for liver cirrhosis, hepatic malignancies, and inherited or acquired thrombophilia (14).

The pathophysiological mechanism increasing the incidence of thromboembolic events has not been fully understood in COVID-19 patients. However, thrombosis associated with inflammation (thromboinflammation) has been described in many diseases, which is due to the loss of normal antithrombotic and anti-inflammatory activities of endothelial cells, leading to the dysregulation of coagulation, complement system activation, platelet activation, and leukocyte recruitment in the microvasculature (15). Any dysregulation in endothelial cells can result in excess thrombin generation and fibrinolysis shutdown, indicating a hypercoagulable state in COVID-19 patients (16).

Contrast-enhanced abdominopelvic CT scan can be the most effective diagnostic tool for splanchnic vein thrombosis. This modality allows us to investigate not only portomesenteric vascular findings (eg, the extent of involved vessels, intravenous contrast filling defects, intraluminal stenosis or occlusion, vascular wall thickening or enhancement, and perivascular fat infiltration), but also enteric findings (eg, bowel infarction, hepatic or splenic infarction, and pneumoperitoneum), which are associated with the patient's death (17).

Additionally, abdominal Doppler ultrasonography can be the primary or alternative diagnostic tool to detect and follow-up splanchnic vein thrombosis, because ultrasound machines have good mobility and accessibility for approaching the patients, especially those who are critically ill and admitted to ICUs (18). However, it has several shortcomings, such as the limited sonic window to investigate the full extent of splanchnic vein thrombosis and lower diagnostic efficiency to identify bowel infarction and pneumoperitoneum. These radiological examinations can help clinicians make prompt and appropriate decisions to choose between a medical treatment, interventional thrombectomy, and surgical intervention at the time of the diagnosis (19).

In conclusion, this is the first case of extensive splanchnic vein thrombosis in a COVID-19 patient from the Middle East. Although splanchnic vein thrombosis is a rare 

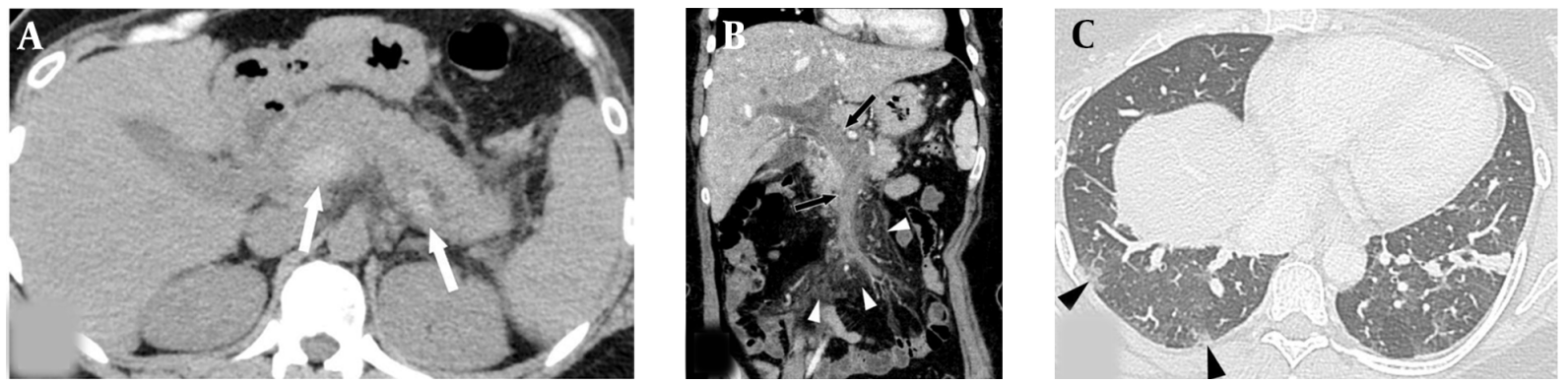

Figure 1. A 43-year-old woman with epigastric pain for one week and a 10-day history of respiratory symptoms confirmed as COVID-19. A, The axial non-enhanced abdominopelvic CT scan shows a voluminous, highly attenuated thrombus in the main portal vein and splenic vein (white arrows). B, Coronal reformated contrast-enhanced abdominopelvic CT scan shows total occlusion, caused by an extensive acute thrombus in the portal and superior mesenteric veins (black arrows) with mesenteric perivascular edema (white arrowheads). C, Axial chest CT scan shows several subpleural lesions with ground glass attenuation in the right lower lobe (black arrowheads).

extrapulmonary complication in COVID-19 patients, clinicians should consider it as one of the differential diagnoses when examining COVID-19 patients with abdominal symptoms, even those without a risk factor for hypercoagulability. Besides, clinicians need to immediately perform radiological examinations, such as enhanced CT scan or abdominal Doppler ultrasonography, for COVID-19 patients with abdominal manifestations.

\section{Footnotes}

Authors' Contribution: Kwang Hwi Lee: Drafting of the manuscript, administrative, technical, and material support, and revision; Eui Yong Jeon: Study conception and design and study supervision and revision; Maryam Hasan Abdulla: Critical revision of the manuscript for important intellectual content; Young Joon Ahn: Critical revision of the manuscript for important intellectual content; George Thomas: Acquisition of data; and Yeong-lee Yu: Analysis and interpretation of data.

Conflict of Interests: The authors declare no conflicts of interest.

Ethical Approval: Ethical approval was not taken for this case report.

Funding/Support: This study received no funding or financial support.

Informed Consent: A consent form was obtained from the patient for this case report. She agreed to the journal's access to her medical history and information related to diagnosis and treatment.

\section{References}

1. Zhang R, Ni L, Di X, Wang X, Ma B, Niu S, et al. Systematic review and meta-analysis of the prevalence of venous thromboembolic events in novel coronavirus disease-2019 patients. J Vasc Surg Venous Lymphat
Disord. 2021;9(2):289-298 e5. doi: 10.1016/j.jvsv.2020.11.023. [PubMed: 33309903]. [PubMed Central: PMC7725061].

2. Franco-Moreno A, Piniella-Ruiz E, Montoya-Adarraga J, Ballano-Franco C, Alvarez-Miguel F, Peinado-Martinez C, et al. Portal vein thrombosis in a patient with COVID-19. Thromb Res. 2020;194:150-2. doi: 10.1016/j.thromres.2020.06.019. [PubMed: 32788107]. [PubMed Central: PMC7293484].

3. de Barry O, Mekki A, Diffre C, Seror M, El Hajjam M, Carlier RY. Arterial and venous abdominal thrombosis in a 79-year-old woman with COVID-19 pneumonia. Radiol Case Rep. 2020;15(7):1054-7. doi: 10.1016/j.radcr.2020.04.055. [PubMed: 32351657]. [PubMed Central: PMC7188660].

4. Li Q, Guan X, Wu P, Wang X, Zhou L, Tong Y, et al. Early Transmission Dynamics in Wuhan, China, of Novel Coronavirus-Infected Pneumonia. N Engl J Med. 2020;382(13):1199-207. doi: 10.1056/NEJMoa2001316. [PubMed: 31995857]. [PubMed Central: PMC7121484].

5. Klok FA, Kruip M, van der Meer NJM, Arbous MS, Gommers D, Kant $\mathrm{KM}$, et al. Incidence of thrombotic complications in critically ill ICU patients with COVID-19. Thromb Res. 2020;191:145-7. doi: 10.1016/j.thromres.2020.04.013. [PubMed: 32291094]. [PubMed Central: PMC7146714].

6. Ofosu A, Ramai D, Novikov A, Sushma V. Portal Vein Thrombosis in a Patient With COVID-19. Am J Gastroenterol. 2020;115(9):1545-6. doi: 10.14309/ajg.0000000000000781. [PubMed: 32694290]. [PubMed Central: PMC7396211].

7. Low SW, Swanson KL, McCain JD, Sen A, Kawashima A, Pasha SF. Gastric ischemia and portal vein thrombosis in a COVID-19-infected patient. Endoscopy. 2020;52(12):E465-6. doi: 10.1055/a-1230-3357. [PubMed: 32877937]. [PubMed Central: PMC7724579].

8. La Mura V, Artoni A, Martinelli I, Rossio R, Gualtierotti R, Ghigliazza G, et al. Acute Portal Vein Thrombosis in SARS-CoV-2 Infection: A Case Report. Am J Gastroenterol. 2020;115(7):1140-2. doi: 10.14309/ajg.0000000000000711. [PubMed: 32618673]. [PubMed Central: PMC7273956].

9. Malik ZR, Razaq Z, Siff M, Sheikh S. COVID-19 Presenting as Banti's Syndrome. Cureus. 2020. doi: 10.7759/cureus.9096.

10. Jafari SH, Naseri R, Khalili N, Haseli S, Bahmani M. Portal vein thrombosis associated with COVID-19: points to consider. Case Rep. 2020;6(3). doi: 10.1259/bjrcr.20200089.

11. Singh B, Kaur P, Maroules M. Splanchnic vein thrombosis in COVID19: A review of literature. Dig Liver Dis. 2020;52(12):1407-9. doi: 10.1016/j.dld.2020.09.025. [PubMed: 33067157]. [PubMed Central: PMC7522621].

12. Ignat M, Philouze G, Aussenac-Belle L, Faucher V, Collange O, Mutter D, et al. Small bowel ischemia and SARS-CoV-2 infection: an underdiagnosed distinct clinical entity. Surgery. 2020;168(1):14-6. doi: 
10.1016/j.surg.2020.04.035. [PubMed: 32473831]. [PubMed Central: PMC7198136].

13. Del Hoyo J, Lopez-Munoz P, Fernandez-de la Varga M, Garrido-Marin A, Valero-Perez E, Prieto M, et al. Hepatobiliary and Pancreatic: A fatal case of extensive splanchnic vein thrombosis in a patient with Covid-19. J Gastroenterol Hepatol. 2020;35(11):1853. doi: 10.1111/jgh.15174. [PubMed: 32839984]. [PubMed Central: PMC7461433].

14. Riva N, Ageno W. Cerebral and Splanchnic Vein Thrombosis: Advances, Challenges, and Unanswered Questions.J Clin Med.2020;9(3). doi: 10.3390/jcm9030743. [PubMed: 32164214]. [PubMed Central: PMC7141239].

15. Jackson SP, Darbousset R, Schoenwaelder SM. Thromboinflammation: challenges of therapeutically targeting coagulation and other host defense mechanisms. Blood. 2019;133(9):906-18. doi: 10.1182/blood-2018-11-882993. [PubMed: 30642917].
16. Varga Z, Flammer AJ, Steiger P, Haberecker M, Andermatt R, Zinkernagel AS, et al. Endothelial cell infection and endotheliitis in COVID19. Lancet. 2020;395(10234):1417-8. doi:10.1016/s0140-6736(20)30937-5.

17. Angelelli G, Scardapane A, Memeo M, Stabile Ianora AA, Rotondo A Acute bowel ischemia: CT findings. Eur J Radiol. 2004;50(1):37-47. doi: 10.1016/j.ejrad.2003.11.013. [PubMed: 15093234].

18. Fortea JI, Puente A, Cuadrado A, Del Pozo C, Garcia I, Pellon R, et al. An Elusive Diagnosis in Noncirrhotic Chronic Portal Vein Thrombosis. Am J Med. 2021;134(3):199-200. doi: 10.1016/j.amjmed.2020.07.039. [PubMed: 32950503].

19. Abeysekera KW, Karteszi H, Clark A, Gordon FH. Spontaneous portomesenteric thrombosis in a non-cirrhotic patient with SARS-CoV2 infection. BMJ Case Rep. 2020;13(12). doi: 10.1136/bcr-2020-238906. [PubMed: 33371000]. [PubMed Central: PMC7757448]. 